\title{
Damages from extreme flooding events to cultural heritage and landscapes: water component estimation for Centa River (Albenga, Italy)
}

\author{
Branka Cuca and Luigi Barazzetti \\ Department of Architecture, Built Environment and Construction Engineering (ABC) Politecnico \\ di Milano, 20133 Milan, Italy
}

Correspondence: Branka Cuca (branka.cuca@polimi.it)

Received: 11 August 2018 - Revised: 19 November 2018 - Accepted: 26 November 2018 - Published: 20 December 2018

\begin{abstract}
The monitoring of hazardous events through change detection has an important role in the emergency management. Such actions can be performed shortly after the hazardous event for first rapid mapping but also over longer periods of time for recovery purposes and risk mapping. The use of medium resolution free-of-charge multi-spectral satellite imagery for purposes of flood extension and impact monitoring can be extremely valuable due to their ability to offer an "easy" and remote access to information, even in cases of extreme weather conditions, but also due to their high compatibility with GIS environments. The case study regards Centa River estuary that hosts an important archaeological site of Albenga within the boundaries of its riverbed. The authors propose a workflow that uses Copernicus Sentinel2 data to provide the comparison changes firstly in the single relevant bands and successively in the indexes NDVI e NDWI, suitable for the estimation of water component. The results of this study were useful for observing the extension of the flooded area, to evaluate its impact on the archaeological remains and to further propose more targeted UAV-born and ground survey.
\end{abstract}

\section{Introduction}

The main damages caused by floods to cultural landscapes and archaeological sites are the loss or potential destruction of landscape elements and its defining features; alteration of landscape functioning (e.g. movements of roadbeds and shorelines, deposits of mud etc.) and deposition of flood debris (Stovel, 1998).
The monitoring activity of hazardous events such as floods has a crucial role in emergency management. In the case of flooding of rivers and torrents, it is important to perform monitoring on the land cover change in the areas bordering the riverbeds and in particular on the effects on the built environment. Such actions can be performed shortly after the hazardous event (first rapid mapping) but also over longer periods of time for recovery purposes and risk mapping (e.g. to investigate possible behavior pattern of the water element observed).

The use of satellite imagery for these purposes can be extremely valuable due to their provision of an "easy" and remote access to information, even in cases of extreme weather conditions, but also due to their compatibility with GIS environments. The added value of satellite-born monitoring regards also the fact that it allows the use of multispectral sensor products, allowing thus not only spatial but also radiometric analysis to be used as input information to the risk-prevention actions. The authors have chosen to consider optical multispectral imagery due to its capacity to provide further possibilities to compare and discuss the results obtained from UAV-born RGB orthophoto imagery. The work presented in this paper is a part of a more comprehensive workflow able to assess damage occurring to heritage sites after extreme floods. This workflow includes satellite, UAV and close-range images used in a multi-scale approach for change detection.

The use of remote sensing images acquired from satellites and UAV platforms is part of a multi-scale approach for change detection. Satellite images allow us to observe a large area, detecting changes at cartographic scale and highlighting critic locations. The use of images acquired with a drone 

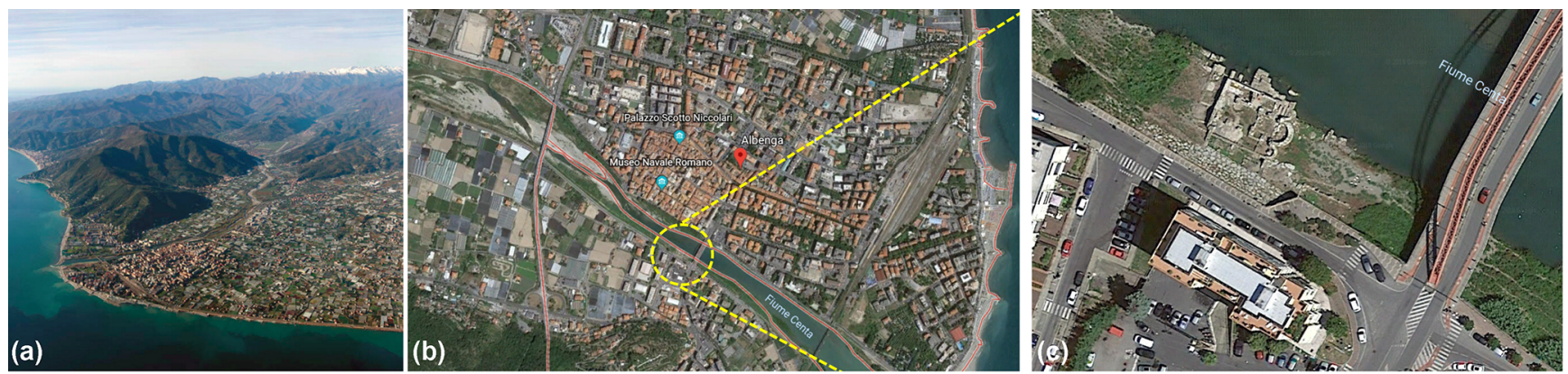

Figure 1. Albenga case study: bird's eye-view of the coast (a); a sequence of the cultural heritage sites in the cultural landscape system of Albenga (b); close-up on the church of St. Clemente archaeological site found in the riverbed of the river Centa.

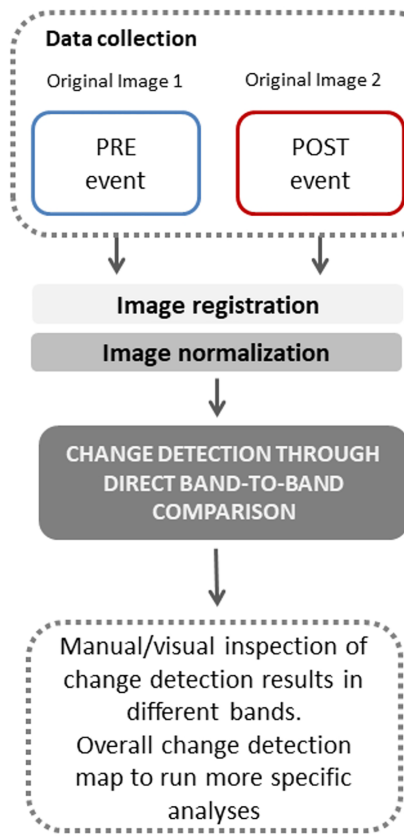

Figure 2. The overview of the workflow proposed.

is the second step. Drone images can be acquired in relatively small areas (if compared to the area captured by satellite images) but they can reveal damages at the level of a heritage site, till the level of small constructive elements.

The case study examined is the unique archaeological site of Albenga, hosted within the boundaries of the Centa riverbed. This site, located in the Liguria region of Italy, is an area composed of significant historic layers: the church of San Clemente, situated on the right bank of the river is mostly out of water, but becomes partially or even totally submerged in great flood events. Such extreme floods exert constant pressure on the structures and on the surrounding landscape, deprived of any kind of protection devices (Fig. 1). Nowadays, this area is still missing an accurate analysis linked with its surroundings and within a context of precarious environmental balance. In November 2016, the site of Albenga suffered serious damages due to a significant flooding event caused by the winter precipitations.

\section{Methodology proposed and datasets used}

The main aim of this work was to investigate the changes in water content caused by the river flooding and affecting the area of the archaeological site. For this purpose two different approaches were considered: (i) on one side change detection through direct band-to-band comparison was performed, (ii) on the other side NDVI and NDWI indexes and their mathematical differences have been calculated before and after the flooding event. The bands considered for bandto-band change detection were band -2 , Band -3 , Band -4 and Band -8 . 
Table 1. Dataset used: Sentinel-2 (S-2) data of Copernicus programme.

\begin{tabular}{ll}
\hline $\begin{array}{l}\text { UAV flight dates } \\
\text { (for comparison purposes) }\end{array}$ & $\begin{array}{l}\text { Sentinel-2 } \\
\text { data }\end{array}$ \\
\hline Before & \\
\hline 23 April 2015 & $\begin{array}{l}\text { No S-2 available yet } \\
\text { 9 October 2016 } \\
\text { 9 November 2016 }\end{array}$ \\
\hline After & \\
\hline $\begin{array}{l}\text { No possibility for performing } 2016 \\
\text { a UAV flight due to unsuitable } \\
\text { weather conditions }\end{array}$ & \\
23 May 2018 & 1 June 2018 \\
\hline
\end{tabular}

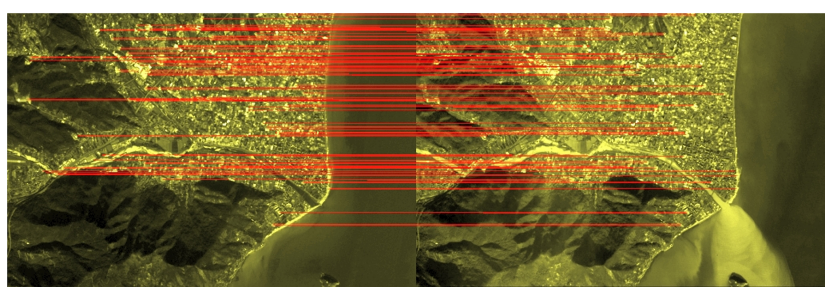

Figure 3. Matched features in both images for the computation of registration parameters. Image contains modified Copernicus Sentinel data (2018).

The use of two different change detection methods is mainly related to the opportunity to highlight changes in the whole area (image difference), followed by a specific change detection analysis caused by floods. Results shown that a combined use of both methods and the integrated inspection using the computed change detection maps provides better results than the use of a single method. The results of the water component estimation were normalized and integrated with other geospatial information (e.g. Region Liguria orthophoto) for further observation. The overall information is envisaged to give some major insight into the behavior of river Centa during and after flooding and to provide qualitative and quantitative inputs to the products required for a more effective emergency management such as rapid mapping, recovery purposes and risk mapping. The overall methodology layout is illustrated in Fig. 2.

A sequence of satellite images was used to analyze the land changes occurring after the last major flooding event that has affected the area in the winter of 2016 (Table 1). A multi-temporal time-series of Sentinel-2 data containing 13 bands in the visible, near-infrared and short wave infrared part of the spectrum was chosen. The data acquired over the Albenga coast in distinct moments before and after the flooding event (occurring between 24 and 25 November 2016) were downloaded from the USGS archive (https: //glovis.usgs.gov/, last access: 12 June 2018). In particular,
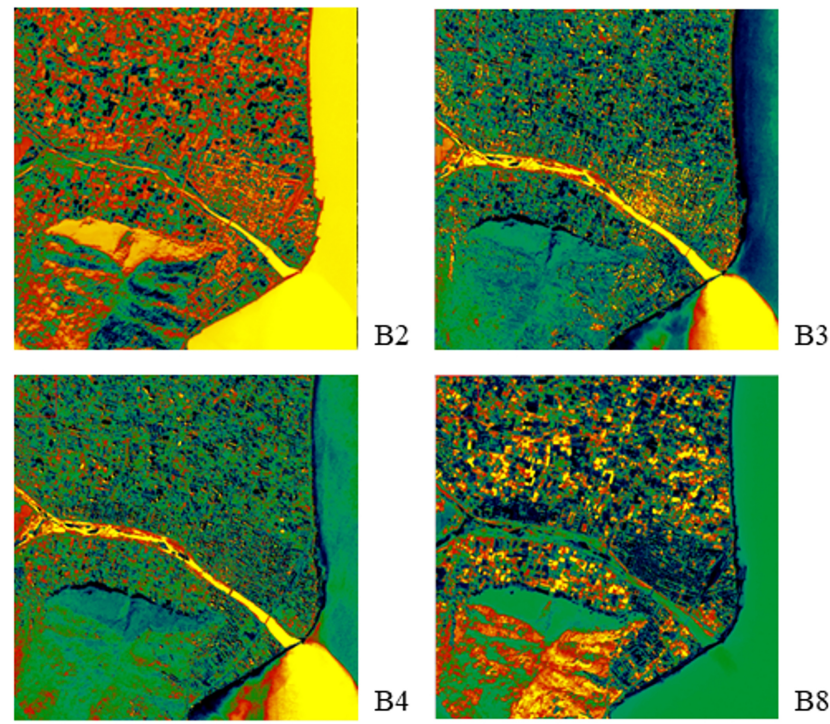

Figure 4. Difference maps computer for four spectral bands with $10 \mathrm{~m}$ geometric resolution. Image contains modified Copernicus Sentinel data (2018).

Table 1 shows spatial and spectral resolution and the acquisition dates of the chosen images and of the specific band examined. Satellite data were selected keeping in mind the minimum percentage of cloud-coverage and choosing the date as close as possible to the dates of UAV flights performed on the site for possible further correlation. Furthermore, it was important to choose images acquired in similar conditions to eliminate variations caused by the different acquisition epochs. In fact, some objects (e.g. vegetation) are constantly subject to changes during the year. Change detection via images acquired in a relatively short time is more robust against such effects. Systematic effects in the change detection can be removed through differencing if the same parameters are applied and images are acquired in a relatively short time.

It is appropriate to mention the meteorological conditions, as reported by the Liguria Regional Agency for Environment Protection (ARPAL). Regarding the conditions of the sea, report highlights that the state of the sea has been moderate for most of the period but on the 24 November it rose to rough. Although the period regarded the winter season, there have been no news of specific damages; however, the news websites have reported about a sea-storm that on the morning of 24 November that has flooded the coastal road between the town of Albenga and the smaller municipality Ceriale located in the north.

In the section "Albenga - Molino Branca" interested by the river Centa (situated in the geographical alert zone A), the increase of the maximum hydrometric level was observed at 14:15 on 24 November 2016 accounting for $5.36 \mathrm{~m}$ causing hence the maximum hydrometric level to reach the total of $5.84 \mathrm{~m}$. (ARPAL Report, 2016). 

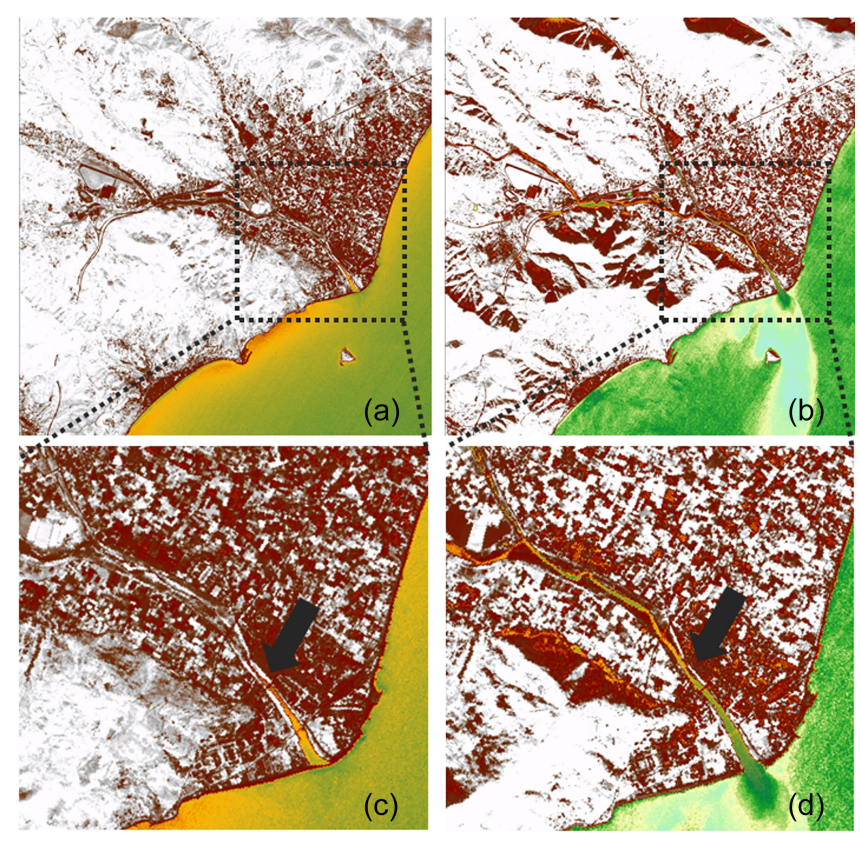

Figure 5. NDVI in false colour gradient calculated over Albenga on 9 October (a) and on 28 November 2016 (b); close up of the Centa river estuary and the inhabited area around the river shores on 9 October (c) and on 28 November 2016 (d). Image contains modified Copernicus Sentinel data (2018).

\section{Change detection through direct band-to-band comparison}

\subsection{Pixel correspondence recovery}

An accurate co-registration between images acquired at different epochs is fundamental to run pixel-based change detection algorithms. Co-registration accuracy for Sentinel-2 images is expected to reach sub-pixel accuracy, as demonstrated in Barazzetti et al. (2016). The method used to validate pixel correspondence is based on the SAMIR project proposed by Gianinetto et al. (2015). SAMIR can match corresponding points in a generic image dataset (two or more images) using the SIFT operator. Outlier rejection is carried out using robust estimators which exploit image geometry, i.e., an affine transformation based on 6 parameters.

Matching was carried using a common band for both images (band 3), obtaining 397 points for each image (Fig. 3). The computation of transformation parameters assumed the first image as a reference, and provided the coefficients for the second one. The small numerical values (e.g., translations of about 0.14 pixels) of transformation parameters confirm the good correspondence of the images, which do not need further data processing for registration. Indeed, such values mainly depends on the accuracy of image matching and appear not to be caused by a slight misalignment of the images.
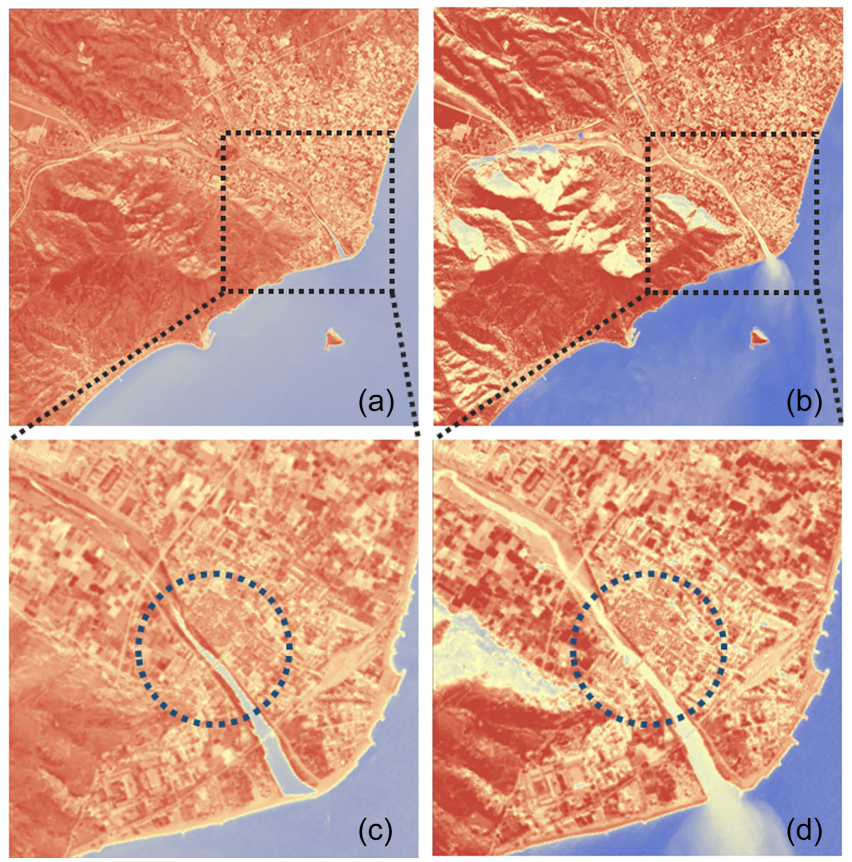

Figure 6. NDWI in false colour gradient calculated over Albenga on 9 October (a) and on 28 November 2016 (b); close up of the Centa river estuary and the inhabited area around the river shores on 9 October (c) and on 28 November 2016 (d). Image contains modified Copernicus Sentinel data (2018).

\subsection{Direct band-to-band comparison}

As images feature pixel-to-pixel correspondence, rapid change detection can be carried out via image differencing. Given two images $I_{1}(x y)$ and $I_{2}(x y)$, image differencing is based on raster algebra following Eq. (1):

$D_{2,1}(x, y)=I_{2}(x, y)-I_{1}(x y)$

It is clear that a small mis-registration error between input images can lead to large errors in the computation of the difference image. Differences can be computed using a specific band, band combinations, or indexes computed from input bands (e.g., NDVI).

After data registration (see previous section) data have been processed to improve change detection results. First, dark object subtraction has been carried out on both images. Then, normalized to a new range between zero and one has been applied to both images. Figure 4 shows the difference maps computed for different spectral bands corresponding to Sentinel-2 bands with $10 \mathrm{~m}$ geometric resolution: B2 $(496 \mathrm{~nm})$, B3 $(560 \mathrm{~nm})$, B4 $(664.5 \mathrm{~nm})$, B8 $(835.1 \mathrm{~nm})$. Differences were computed for all bands and a colorscale was then applied to visualize changes. It is interesting to notice that the river area features large differences in band 3 and 4 . This still hold when the river flows into the sea.

As mentioned, only image differencing has been tested instead of using more advanced change detection algorithms 

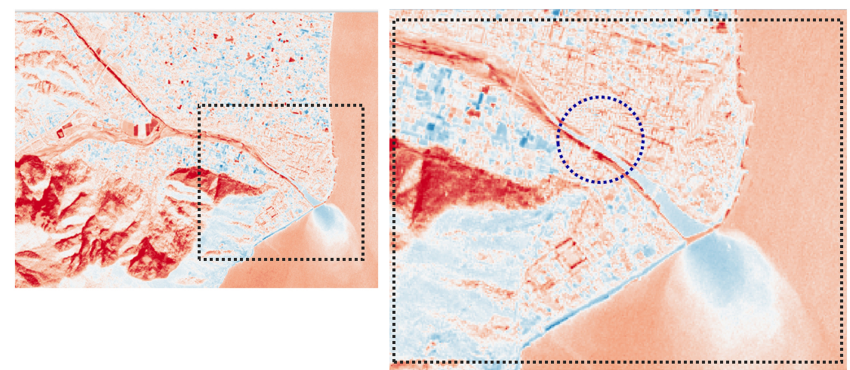

Figure 7. Mathematical difference of NDWI value over town of Albenga between dates 9 October and 28 November 2016. Image contains modified Copernicus Sentinel data (2018).

available in commercial software packages for remotely sensed data processing. This option was chosen due to several reasons. Firstly, images were captured by the same satellite, so data are acquired in the same range of the spectrum, with the same band wavelength and same response function. In addition, Dark-Object Subtraction (DOS) has also been performed to limit the differences in atmospheric conditions. Finally, images were acquired in a period of a couple of month (9 November 2016/28 November 2016) in which limited changes are expected at least for urban areas. Vegetated areas are instead much more sensitive to the different acquisition epoch.

Although very fast and straightforward, image difference based on different bands cannot distinguish between changes caused by different factors, such as vegetation changes or changes due to muddy flood. As the aim of this work is to investigate water content caused by the river, a procedure for change detection based on a combination of NDVI and NDWI has been developed, as illustrated in the following section.

\section{Change detection using NDVI and NDWI indexes: results and comparison}

\subsection{Change detection of the water component in Albenga area using NDVI and NDWI}

In the second approach the two multi-spectral Sentinel-2 images were used to calculate the Normalised Difference Vegetation Index (NDVI) values of the case study area in two distinct dates before ( 9 October) and just after the 2016 flooding event (28 November). This index was chosen for some first observation as the water has a very low reflectance in both wavelengths used by NDVI (NIR and red), building up on the work conducted in (Cuca, 2017). Figure 5 illustrates the index calculated for the municipality area of Albenga with a close-up on the estuary of the river Centa. The black arrow indicates the area near the Emidio Viveri bridge (known as "red bridge") in which vicinity the St. Clement church remains are located.
The image of NDVI was reclassified in false colours to facilitate a visual inspection: it can be easily noted that the area covered by water (yellow and green) significantly increases in the width after the seas-storm, approaching thus the shore of the riverbed and probably covering all the archaeological remains.

Another inspection was conducted using Normalised Difference Water Index (NDWI) to monitor changes related to water content in water bodies, using green and NIR wavelengths; as defined by McFeeters (1996), in this case, water features have positive values and are thus enhanced. The index was calculated applying the following Eq. (2):

NDWI $=($ XGREEN - XNIR $) /($ XGREEN + XNIR $)$

The results illustrated in Fig. 6 illustrate the index calculated for the municipality area of Albenga with a close-up on the estuary of the river Centa with focus on the area of the archaeological remains (blue circle). Again, the image of was reclassified in false colours to facilitate a visual inspection: it can be noted easily that the area covered by water (blue and yellow colour) has significantly increased in the width, again approaching the shore of the riverbed endangering the archaeological remains present.

To explore better the nature of change, a mathematical difference between two images has been computed resulting in an image presented in a Fig. 7, re-proposing the method experimented in (Cuca and Agapiou, 2017). On a blue-red colour scale (with blue indicating minimum changes occurring and red signalling the maximum ones), it can be observed how a quite significant change has been exerted on the south-western shore in proximity of the "red bridge" between 9 October and 28 November 2016.

\section{Discussion of work}

The work here illustrated proposes to perform change detection using medium resolution multi-spectral satellite imagery through both band-to-band comparison and through index comparison used for water component extraction.

An advantage of the band-to-band approach is that it is easy and straight-forward and it provides an immediate insight into major changes. However, the image difference based on different bands cannot distinguish between changes caused by different factors, such as vegetation changes or changes due to muddy flood. Other indexes such as NDVI and especially NDWI for detection of water component in water bodies, give results that provide much clearer interpretation. One disadvantage of NDWI seems to be the presence of "shadow" areas that can cause some problems during the interpretation phase. To overcome these issues, a vector layer could be used as a buffer so as to consider only the area of interest. Furthermore, a tresholding procedure could give even better results providing distinct classes for water features and other classes, potentially enabling the calculation of quanti- 


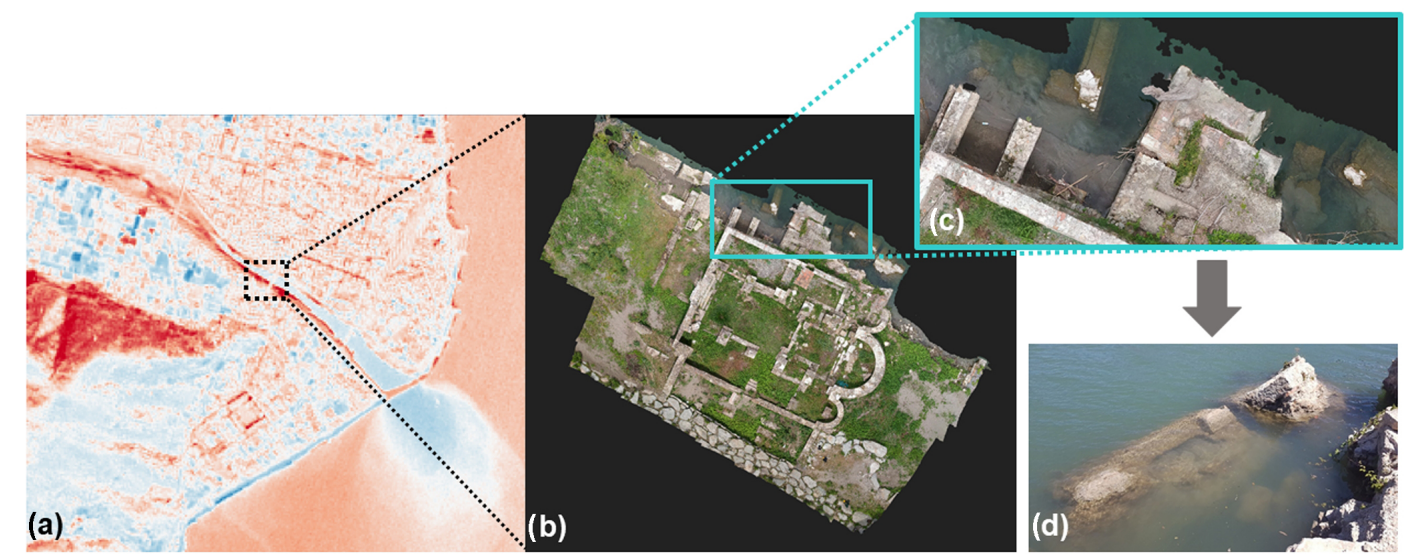

Figure 8. A proposal for a survey workflow including optical multi-spectral and RGB data at different scales: satellite imagery at $10 \mathrm{~m}$ resolution (a); UAV-born orthophoto of the remains pf St. Clement church, elaborated for a scale 1: 100 (b) and close-up (c); photo documentation of the damage visible from the ground caused by the flooding event in November 2016 (d).

tative results in terms of exact area affected by the changes (i.e. quantitative estimation of the flooded area).

It is important to mention that radar technology would have been a more suitable option to consider, given also the weather conditions, as already proposed by literature (Pulverini et al, 2011; Vasileva et al., 2015; Zoka et al., 2018). However, the aim of this work was to propose a direct connection between optical multi-spectral and RGB data at different levels starting from satellite $10 \mathrm{~m}$ resolution imagery arriving to UAV-born orthophotos at few centimeters terrain pixel resolution.

In this way, satellite data could be processed systematically so as to provide a kind of pre-alert service that would trigger further focused survey from UAVs (with possibility of including targeted terrestrial equipment), in a more integrated workflow (Fig. 8). In addition, UAV and terrestrial surveys could be proposed as a planned service for monitoring and validation once a year. The integration of the two approaches is of a crucial importance for the case of Albenga archaeological site for two reasons: (i) to evaluate the extension and impact of flooding on a macro-level (territorial scale) and (ii) to provide valuable information on the state of the art of archaeological assets found within the riverbed area to the local superintendence (building/feature scale). Closer observation of different episodes of the flooding phenomena might give better indications regarding the preventive maintenance measures that would be suitable in the specific case of St. Clemente church remains.

\section{Conclusions}

This paper illustrated an approach to evaluate damages after extreme flooding, with special attention to cultural heritage conservation. The paper mainly focuses on the use of Sentinel-2 (optical) satellite images with medium geomet- ric resolution, which are available at specific epochs without purchasing high resolution images on the selected area. The idea behind this paper is to consider damage identification as a change detection problem, which starts from the satellite level and reaches the local level using data acquired from drones and terrestrial images, i.e. through a multi-scale approach, as illustrated in the previous section.

The work carried out is mainly related to processing of satellite images, which can be used to plan the acquisition of on-site data (UAV and close-range photogrammetry). Change detection was carried out with both (i) traditional algorithms based on image differencing, and (ii) a novel approach that tries to focus on water body mapping using NDVI and NDWI. Results reveal that image difference is a valid tool for rapid analysis, which however provides an overall change detection for the entire area. The inspection around the river area require manual interpretation of the maps estimated via differencing, obtaining more qualitative than quantitative results.

The estimation of NDWI proved to be a more robust index for the river area, obtaining a change detection that can better follow the variations occurred in the riverbed. On the other hand, NDWI produces false variations also for other elements visible in the images, especially for area with shadows, such as the mountain around Albenga. These effects can be easily filtered oud and corrected using additional information from geospatial databases, e.g. adding a river layer and limiting the analysis to a specific buffer area generated around a selected feature.

Future work consists in the acquisition and processing of UAV and close-range images starting from information collected from satellite images. Although specific elements of the sites affected by flooding are not visible in the medium resolution images, the outcomes of the satellite-change detection workflow can be used to plan image work on-site, especially the geographic locations where major damages are 
expected as well as the time frequency for data collection on site.

Data availability. Free, full and open data access to Sentinel images is available at the link https://scihub.copernicus.eu/dhus/\#/ home (last access: 17 December 2018).

Competing interests. The authors declare that they have no conflict of interest.

Special issue statement. This article is part of the special issue "Earth surveillance and space-based monitoring of the environment: integrated approaches". It is a result of the EGU General Assembly 2018, Vienna, Austria, 8-13 April 2018.

Acknowledgements. This work has been partially supported by the GAMHer project: Geomatics Data Acquisition and Management for Landscape and Built Heritage in a European Perspective, PRIN: Progetti di Ricerca di Rilevante Interesse Nazionale - Bando 2015, Prot. 2015HJLS7E. The Authors acknowledge the Copernicus Programme for distribution of free, open and full Sentinel-2 data. The articles contains modified Copernicus Sentinel data (2018). The Authors would also like to thank the Superintendence of Metropolitan city of Geona (Soprintendenza Archeologia Belle Arti e Paesaggio per la Citta' Metropolitana di Genova e le province di Imperia, La Spezia e Savona) for granting the access to the site during terrestrial survey fieldwork and for their precious feedback.

Edited by: Silas Michaelides

Reviewed by: Oriel Prizeman and one anonymous referee

\section{References}

Agency for Environmental Protection of the Liguria Region (ARPAL): Report on the meteo-hydrological event occurring during 20-25/11/2016 (authors' translation), edited by: Arena N., Turato B., Soatto F., and Giannoni F., (Rapporto di evento meteoidrologico del 20-25/11/2016), available at: https://www. arpal.gov.it/contenuti_statici//pubblicazioni/rapporti_eventi/ 2016/REM_20161121-25AllertaRossa_vers20170217.pdf (last access: 3 August 2018), 2016.
Barazzetti, L., Previtali, M., and Cuca, B.: Evaluation of registration accuracy between Sentinel-2 and Landsat 8. Proc. SPIE 9688, Fourth International Conference on Remote Sensing and Geoinformation of the Environment (RSCy2016), 4-8 April, Paphos, Cyprus, 9 pp., 2016.

Cuca, B.: THE CONTRIBUTION OF EARTH OBSERVATION TECHNOLOGIES TO MONITORING STRATEGIES OF CULTURAL LANDSCAPES AND SITES, Int. Arch. Photogramm. Remote Sens. Spatial Inf. Sci., XLII-2/W5, 135-140, https://doi.org/10.5194/isprs-archives-XLII-2-W5-135-2017, 2017.

Cuca, B. and Agapiou, A.: IMPACT OF LAND USE CHANGE TO THE SOIL EROSION ESTIMATION FOR CULTURAL LANDSCAPES: CASE STUDY OF PAPHOS DISRICT IN CYPRUS, Int. Arch. Photogramm. Remote Sens. Spatial Inf. Sci., XLII-5/W1, 25-29, https://doi.org/10.5194/isprs-archivesXLII-5-W1-25-2017, 2017.

Gianinetto, M., Barazzetti, L., Dini, L., Fusiello, A., and Toldo, R: Geometric registration of remotely sensed data with SAMIR, Proc. SPIE 9535, Third International Conference on Remote Sensing and Geoinformation of the Environment (RSCy2015), 95350Q, 16-19 March, Paphos, Cyprus, https://doi.org/10.1117/12.2192424, 9 pp., 2015.

McFeeters, S. K.: The use of Normalized Difference Water Index (NDWI) in the delineation of open water features, Int. J. Remote Sens., 17, 1425-1432, 1996.

Pulvirenti, L., Chini, M., Pierdicca, N., Guerriero, L., and Ferrazzoli, P.: Flood monitoring using multi-temporal COSMOSkyMed data: Image segmentation and signature interpretation, Remote Sens. Environ., 115, 990-1002, 2011.

Stovel, H.: Risk preparedness: A management manual for World Cultural Heritage, Rome: ICCROM, 1998.

Vassileva, M., Nascetti, A., Giulio Tonolo, G., and Boccardo, P.: Unsupervised flood extent detection from SAR imagery applying shadow filtering from SAR simulated image, 2015 IEEE International Geoscience and Remote Sensing Symposium (IGARSS), Milan, 2707-2710, https://doi.org/10.1109/IGARSS.2015.7326372, 2015.

Zoka, M., Psomiadis, E., and Dercas, N.: The Complementary Use of Optical and SAR Data in Monitoring Flood Events and Their Effects, Proceedings 2018, 644, 2018. 